\title{
Mandibular Fractures in Different Pediatric Age Groups and their Current Management Modalities
}

\author{
MU AKHTAR MR CHATHA A A SHAH MAUHAQ* \\ Department of Oral \& Maxillofacial Surgery, de' Montmorency. College of Dentistry, Lahore. \\ * Department of Anaesthesia. Children Complex, Lahore \\ Correspontence to Dr. Muthummat lsman. Akhtar. Assistant Professor E mail: alatechahatmail.com
}

Background: A number of cases of growing age trauma within oro-facial region have been observed in Pakistan, unlike developed countries of world. Mandibular fractures occur in greater frequency than those of the middle third of the face in children, later being very small and plastic comparative to mandible. These fractures of mandible do not demonstrate different clinical features as compared to adults but there is relative difference in treatment management due to, different patterns and plasticity of the facial bones in children, developing tooth buds at different levels in the jaws, mixed dentition, shedding deciduous teeth and incomplete roots of anterior teeth. The causes and patterns of fracture in children older than 12 years resemble to those found in adults. Mandibular fractures are common in Pakistan and commonly related etiology is accidental fall. Method: 57 patients presented with mandibular fractures at Oral and Maxillofacial Surgery department of Punjab Dental Hospital and Children Complex Hospital, Lahore from September 2003 to September 2005. The age of the patients ranged from infancy to early teenage ( 6 month to 15 years). After confirming diagnosis, the children were divided into following four groups; Group "A" (Infants), Group "B" (Pre-school), Group "C” (School) and Group "D” (Teenage). All patients were treated with one of the following treatment modalities, depending upon the site of fracture and age group of the patients; Micro and miniplates, Eric arch bar splints, Acrylic splints, Ivy eyelet wires, Trans-osseous wiring and Conservative treatment. Results: Group "A" of only 3 patients was managed with open reduction and microplate rigid fixation. Six months follow up showed healing without any complications. Group 'B' with $60 \%$ males, had mostly body fractures, was treated with acrylic splints and circummandibular wires. Two bilateral body fractures, with avulsed few teeth, were fixed with rigid fixation. Five patients of about age 3 with minimally displaced body fractures were managed with Eric arch bar fixation alone. Two bilateral condylar bowing fractures were treated conservatively (no active treatment) with no TMJ complications during follow up. The mandibular trauma was noticed exceedingly high in group ' $\mathrm{C}$ ' with overall $50.87 \%$ and exclusively in males (90\%). Acrylic splints were utilized to treat either condyle with body fractures or condyles alone. Two children had postoperative ipsilateral jaw deviation on opening, which was improved with reverse elastic exercises during follow up. Five patients of high condylar fractures with either no or minimum occlusal disturbance, were conservatively managed. Early mobilization was the prime concern in them to avoid late complications. $17.54 \%$ patients were of group ' $D$ ' with again high male (90\%) ratio. Two horizontally unfavorable angle fractures were managed by trans-osseous wires. Five with minimum displacement were managed with miniplates rigid fixation. All others' with associated condylar fractures were treated with Eric arch bar splint with one plate at body and intermaxillary fixation for two weeks. Conclusion: The patterns and management modalities of pediatric mandibular fractures vary in different age groups. The pediatric fractures should be managed as early as possible to avoid complications. Majority of trauma results in school going and teenage groups with definite high male proportion. High condylar fractures should be treated with extra care, keeping in mind of post traumatic TMJ ankylosis. Acrylic splints showed ideal results in body and condylar fractures, whereas, microplates may be the treatment of choice in infants with no or few deciduous teeth. No serious complications were observed during 6 months follow up.

Key words: Trauma, mandibular fracture, rigid fixation, splints, intermaxillary fixation and jaw opening exercises.

Mandibular fracture is the break in its continuity, involving single or both cortical plates, confirmed clinically and radio-graphically.

Facial trauma at growing age is relatively common in Pakistan.' The mandibular fractures occur in greater frequency than those of the middle third of the face, later being very small and plastic comparative to mandible at this age $e^{2.3}$. The pediatric mandibular fractures do not demonstrate different clinical features comparative to adults but there is relative difference in treatment plans ${ }^{4-6}$.

The socio-economic status in underdeveloped countries has strong impact at the outcome of the instant trauma to the children particularly in the poorly assessed areas of villages. Unavailability of trained specialists in these areas is again a psychic dilemma. Accidental fall (trees, stairs and buildings), Road traffic accidents (RTA) and assaults trauma are the leading causes for the fracture of mandible in adults ${ }^{1.7}$. In children during road traffic accidents and falls, the head and face are the most vulnerable 2.8 .9 . Anatomically, fractures of mandible may present at alveolar process, symphysis, parasymphysis, body, angle, condylar, coronoid process and combination of any of the above ${ }^{10}$. Condylar region is commonly involved, followed by the parasymphyseal and angle, respectively ${ }^{10,11}$

The management of mandibular fractures in children is different from that of adults as the former exhibits much more liability to emergency management, greater difficulty

in

p

\section{${ }_{(\mathrm{P})}^{\mathrm{pa}}$}

tal

\section{$\mathrm{r}$}

\section{fo}

(

G 
in clinical and radiological examination. state of mixed dentition, faster rate of healing and concomitant intracranial \& cervical spine injuries ${ }^{11.12}$. The treatment ranges from conservative to close and open reduction with or without fixation. The close reduction is favored whenever possible as open reduction either limits or interrupts the osteogenic potential of the periosteum which results in scar formation hence further restricting the growth in the children.' ${ }^{2}$ Rigid fixation is favored whenever open reduction is the choice. The resorbable plates are recent development, alternative to titanium plates. ${ }^{12.13}$ Commonly observed late complications are the post-traumatic temporomandibular joint (TMJ) ankylosis and facial asymmetry, in these patients ${ }^{1,14.15}$.

The adult mandibular fractures have been studied broadly in local literature but not much literature is available about the pediatric mandibular fractures. The purpose of the study is to determine the management options of mandibular fractures and to restrict the posttraumatic late complications, in the different pediatric age groups.

\section{Materials and methods:}

In a descriptive case series study, fifty-seven patients of both gender presented with mandibular fractures at Oral and Maxillofacial Surgery department of Punjab Dental Hospital and Children Complex Hospital, Lahore from September 2003 to September 2005. The age of the patients ranged from infancy to early teenage $(6$ month to 15 years).

The diagnosis was established on the basis of history, clinical and radiographic examination. The standard orthopantomograph (OPG) was taken of every possible patient and other views such as posterio-anterior mandible (PA), lateral oblique and in few cases CT scans were also taken. After hospitalization and necessary investigations, all patients were treated under general anesthesia as random mandibular fractures. Data concerning age, gender, cause, site of fracture and treatment options, was collected. The age of the children was divided into following four groups;

Group "A" (Infants) from $>6$ months to 2 years.

Group "B" (Pre-school) from $>2$ years to 4 years.

Group "C" (School) from $>4$ years to 12 years.

Group "D" (Teenage) from $>12$ years to 15 years.

All patients were treated with one of the planned treatment modalities depending upon the site of fracture and age groups. Patients with pathological fractures, pan- facial fractures and mal-united treated fractures were excluded in the study. Close and open reduction both treatment options selective to the site of the fracture, were adapted. Ivy eyelets with 0.35 gauge soft surgical steel wire were passed between the teeth followed by intermaxillary fixation. The Eric arch bar splint was fixed on the teeth with 0.35 gauge wire after reducing the segments. 0.45 gauge wire was used to stabilize the acrylic splints, which were fabricated after assembling the models from segmental impressions and model surgery. Upper jaw models were used as fixed reference. Titanium micro plates of $1.1 \mathrm{~mm}$ and mini plates of $1.5 \mathrm{~mm}$ and $2.0 \mathrm{~mm}$ were adapted with self threaded $4 \mathrm{~mm}$ and $6 \mathrm{~mm}$ length titanium screws, respectively.

The wooden spatulas exercise, chewing gums and massage (pre-auricular) were recommended to condylar fracture patients to avoid ankylosis of TMJ. Patients were assessed initially weekly for 4 weeks followed by fortnightly for 8 weeks, postoperatively. The intermaxillary fixation (IMF), when performed, was restricted for 2 to 3 weeks and similar was in cases treated with arch bar splints.

The presenting age groups and gender are shown in table 1 whereas the treatment modalities are plotted in graph 1.

\section{Graph 1: Treatment modalities}

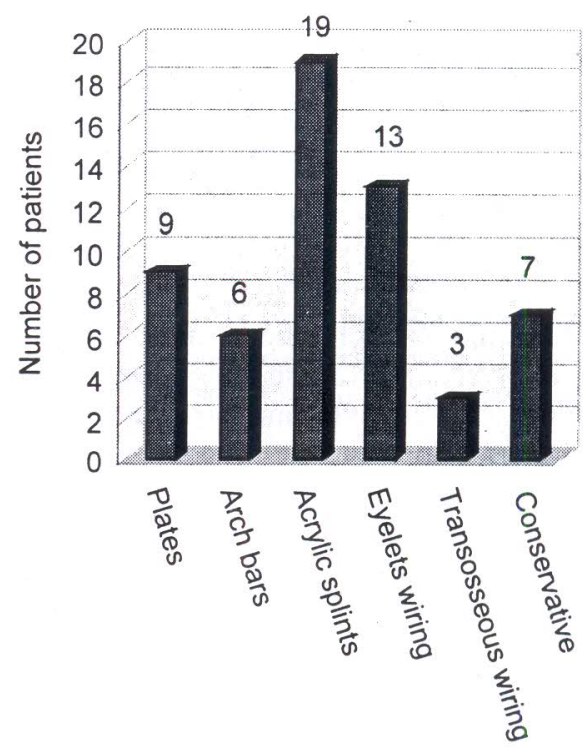

Table: 1: Age Groups, Gender \& Percentage of the Patients in Pediatric Mandibular Fractures:

\begin{tabular}{llllll}
\hline Groups & Age & Males & Females & Total & Group\% \\
\hline A (Infants) & 6 months to 2year & 2 & 1 & 3 & 05.26 \\
B (Pre-school) & $>2$ to 4 years & 9 & 6 & 15 & 26.31 \\
C (School) & $>4$ to 12 years & 26 & 3 & 29 & 50.87 \\
D (Teenage) & $>12$ to 15years & 9 & 1 & 10 & 17.54 \\
\hline
\end{tabular}




\section{Results:}

$5.26 \%$ (3) patients of total being studied were from group 'A' with male to female $2: 1$ ratio and the cause of the trauma narrated by parents of these children was fall from beds or hands. All of these patients had displaced fractures at the symphysis with partially erupted deciduous incisors and they were being bottles. feed too. Single five holes microplate $(1.1 \mathrm{~mm})$ using $4 \mathrm{~mm}$ titanium screws after open reduction was adapted to fix the fractured fragments at the lower border in each patient. Post-operative follow up of six months showed healing without any complications. The parents were asked to resume bottle feeding soon after the rigid fixation.

Group 'B' was 26.31\% (15) patients with $60 \%$ males. These toddlers had just started moving around and were more prone to accidents, particularly fall from stairs and slipping on the floor. These patients frequently had body fracture and were treated with acrylic splints stabilized by circummandibular wiring. Two cases had bilateral body of the mandible fracture with few avulsed teeth and they were fixed with open reduction and rigid fixation by mini-plates $(1.5 \mathrm{~mm})$ likewise in group 'A'. Five patients of age about 3 years with minimum displaced body fractures were managed with Eric arch bar fixation in the lower jaw only. Two patients with bilateral condylar bowing were treated conservatively (no treatment) and found remodeling at fracture site without mal-union or TMJ complications during 6 months of follow up. These patients had adequate postoperative inter-incisal jaw opening, whereas wooden spatulas exercises were advocated to achieve the desirable resuits.

The mandibular trauma was exceedingly high in the school going patients (group ' $\mathrm{C}$ ') measuring overall $50.87 \%(29)$ with male $(90 \%)$ predominance. The causes included RTA (bicycle ride) and fall from height (kite flying \& tree climbing) and body of the mandible was commonly involved site. Acrylic splints were placed in the patients either with condyle and body fractures or only condyle fractures with the goal to achieve the maximum inter-incisal jaw opening during follow up and thus avoiding TMJ ankylosis. The results were highly promising with no TMJ ankylosis during 6 months follow up. Two children had postoperative deviation of the jaw on mouth opening towards condylar fracture side and subsequent improvement was seen with use of reverse elastics. The patients with associated bilateral condylar fractures were exclusively treated with occlusal acrylic splints considering that they would help in raising the posttraumatic posterior facial height and thus prevent the fracture site from haemotoma organization which contributes in fibrosis and TMJ ankylosis later on. Five patients with high condyle fractures with either no or minimum occlusal disturbance were managed conservatively. The early mobilization of the jaw was prime consideration in them to prevent future complications.
$17.54 \%$ (10) was group 'D' (teenage) and data analysis showed extra ordinary high males $(90 \%)$ ratio. The patterns of fracture in these patients were either at symphysis and angle or body and condyle combined. The causes of trauma were intrapersonal violence, RTA and fall from height during kite flying. Two horizontally. displaced fractures at the angle were managed by transosseous 0.5 gauge soft surgical wires and five without displacement at angle were managed with $2 \mathrm{~mm}$ titanium mini-plates fixed with $6 \mathrm{~mm}$ single cortex self threaded screws at angle and parasymphyseal areas of mandible. Patients of body fractures with associated condylar were treated with Eric arch bar splints on both jaws and one miniplate at body fracture area along with intermaxillary fixation for only two weeks. The lower jaw splint was removed after 4 weeks.

\section{Discussion:}

The patterns of mandibular fractures in children vary due to specific habits and socio-economic status of the patients. In the countries of Indian Subcontinent such as Pakistan, the tree climbing, kite flying and small multistory houses are the common associated factors in receiving early age trauma and its consequences are more severe in the form of ankylosis of TMJ and facial asymmetry. Mal-union at fracture site is usually evident by the time patient reaches the tertiary centre for management whereas, in developed countries, the RTA and child abuse are common reasons.

The age group data analysis shows that the mandibular trauma is exceedingly high in school (group 'C') showing 50.87\% (29) of all patients with $90 \%$ male predominance.

We used different modalities to treat the fracture in these children at various age groups. The infants (3) were treated with micro-plate osteosynthesis and the reason of the choice was the presence of only two to four partially erupted anterior deciduous teeth along with extremely delicate mucosa. Moreover, these infants were being feed by the bottle and heavy pressure against the displaced fragments during sucking allowed no other choice except open reduction and rigid fixation. Rigid osteosynthesis results in immediate postoperative functioning of jaw and is an effective and predictable alternative for the treatment of fracture of the mandible ${ }^{16}$.

The cases with parasymphysis along with condylar fractures were treated with occlusal acrylic splints. This choice facilitated early mobilization of the joint and this alleviated the chance of postoperative TMJ ankylosis. The occlusal acrylic splint resulted in excellent healing at symphysis and parasymphyseal areas and they were placed maximum for three weeks. Conservative treatment of condylar fractures in children results in satisfactory long term outcome, despite a high frequency of radiological aberrations, soft tissue diet and immediate immobilization seems to be the treatment of choice ${ }^{17}$. 
Mandibular angle fractures were treated by either mini-plates or trans-osseous wiring. The oblique and horizontally displaced fractures were treated with transosseous wiring in teenage group along with inter-maxillary fixation. The males were dominant victims and common site was angle of the mandible which was reduced with open reduction. The rigid fixation was preferred in bilateral fractures of " $C$ " and " $D$ " groups, while no intermaxillary fixation was performed in them. The mini-plate osteosynthesis has the advantage over conventional intermaxillary fixation. ${ }^{18}$

Arch bar splints were fixed on the lower teeth in " $B$ " group, where there were no condylar fractures without inter-maxillary fixation. This group had maximum deciduous teeth in the jaw along with immature masticatory apparatus. Arch bars were removed after two weeks with excellent healing at the fracture site. One patient had displaced arch bar during immediate follow up which was re-fixed on the next day with extra care and satisfactory post operative healing was observed. Bilateral condylar fractures accompanying symphyseal fractures were managed with acrylic splints. This treatment was carried out in school group exclusively to prevent the complication of ankylosis during subsequent time. Condylar fractures (intra-capsular or extra-capsular) cause direct damage to TMJ bones as well as result in disturbances of dentofacial development later on. ${ }^{19}$ Conservative treatment will nevertheless be sufficient in case of minor displacement due to higher ability of remodeling even more so in children this trend was also prompted when surgeons learned that condyles determine local growth but does not serves as growth center of the mandible. $^{20}$

The patients with isolated condylar fractures, unilateral or bilateral, had been managed without surgical intervention and only jaw exercises had been advocated to keep the mouth functioning and observed for 6 months for the interincisal jaw opening. The results were highly encouraging whereas only two patients of unilateral condylar fracture were observed with jaw deviation towards the affected site during opening of the jaws. The deviation was improved with the use of reverse elastics. The mean interincisal opening was $42 \mathrm{~mm}$ in them. Non-surgical treatment of condylar fractures in children results in satisfactory longterm outcome of the jaw function, despite the relative high frequency of radiologically noted aberrations. ${ }^{21}$

\section{References:}

1. Abbas I, Ali K, Mirza YB. Spectrum of mandibular fractures at a tertiary care dental hospital in Lahore. J Ayub Med Coll Abottabad 2003; 15: 12-4.

2. Kubiak R, Slongo T. Unpowered scooter injuries in children. Acta Pediatr 2003; 92: 50-4.
3. Defabianis P. TMJ fractures in children and adolescents: Treatment guidelines. J Clin Pediatr Dent 2003; 27 : 191-9.

4. Dongas P, Hall GM. Mandibular fracture patterns in Tasmania, Australia. Aust Dent J 2002; 47: 131-7.

5. Lin $\mathrm{Y}$, Wang $\mathrm{X}, \mathrm{Yi}$ B. Miniplates osteosynthesis of mandibular fractures. Zhonghua Kou Qiang Yi Xue Za Zhi 2001; 35: 85-7.

6. Chidyillo SA, Jacobs JS. Applications of dental splints with regard to modern techniques of rigid fixation. J Craniofac Surg 1994; 5: 136-41.

7. Rasse M. Recent developments in therapy of condylar fractures of the mandible. Mund Kiefer Gesichtschir 2000; 4: 69-87.

8. Haug RH, Foss J. Maxillofacial injuries in the pediatric patients. Oral Surg Oral Med Oral Pathol Oral Radiol Endod 2000; 90: 126-34.

9. Gassner R, Tuli T, Hach O, Rudisch A, Ulmer $H$. Crainomaxillofaical trauma: a 10 year review of 9543 cases with 21067 injuries. J Cranomaxillofac Surg 2003; 31: 5161.

10. Iidor S, Matsuya T. Pediatric maxillofacial fractures: their aetiological characters and fracture patterns. J Craiomaxillofac Surg 2002; 30: 237-41.

11. Hussain S, Ahmad M, Khan M, Anwar M, Amin M et al Maxillofacial trauma: current practice in management at Pakistan Institute of Medical Sciences. J Ayub Med Coll Abottabad 2003; 15: 8-11

12. Grasser R, Tuli $T$, Hachl $O$, Moreeira $R$, Ulmer $H$. Crainomaxillofacial trauma in children: a review of 3385 cases with 6060 injuries in 10 years. J Oral Maxillofac Surg 2004; 62: 399-407.

13. Kaban LB. Dentoalveolar injuries and mandibular fractures in: Kaban LB. Pediatric maxillofacial surgery. Philadelphia: WB Saunders 1990; 233-260.

14. Abbas I, Ali K. Management of mandibular fractures. Pak Oral Dental J 2002; 22: 151-4.

15. Hoffmeister B. Pediatric maxillofacial trauma. In: Booth PW, Schandel SA, Housaum JE. Maxillofacial surgery. London: Churchill Livingstone 2000; 221-228.

16. Ardary WC. Prospective clinical evaluation of the use of compression plates and screws in the management of mandibular fractures. J Oral Maxillofac Surg.1989; 47: 1150-3.

17. Girthofer K.Goz G. TMJ remodeling after condylar fractures and functional jaw orthopedics a case report. J Orofac Orthop. 2002; 63: 429-34.

18. Koltai PJ, Rabkin D, Hoehn J. Rigid fixation of maxillofacial fracture in children. J Craniomaxillofac Trauma. 1995; 1: 32-42.

19. Thoren H, Hallikinen D, lizuka T, Lindqvist C. Condylar process fractures in children: a follow-up study of fracture with total dislocation of the condyle from the glenoid fossa. J Oral Maxillofac Surg. 2001; 59: 768-73; discussion 773-4.

20. Remi M, Christine MC, Geal P, Soizik P, Josep-Andre J. Mandibular fractures in children: long term results. J Pediatr Otorhinolaryngol. 2003; 67: 25-30.

21. Chen J, Oh N, Kim I. A follow-up study of condyle fracture in children. J Oral Maxillofac Surg. 2005; 34: 851-58 
Mandibular Fractures in Different Pediatric Age Groups and their Current Management Modalities

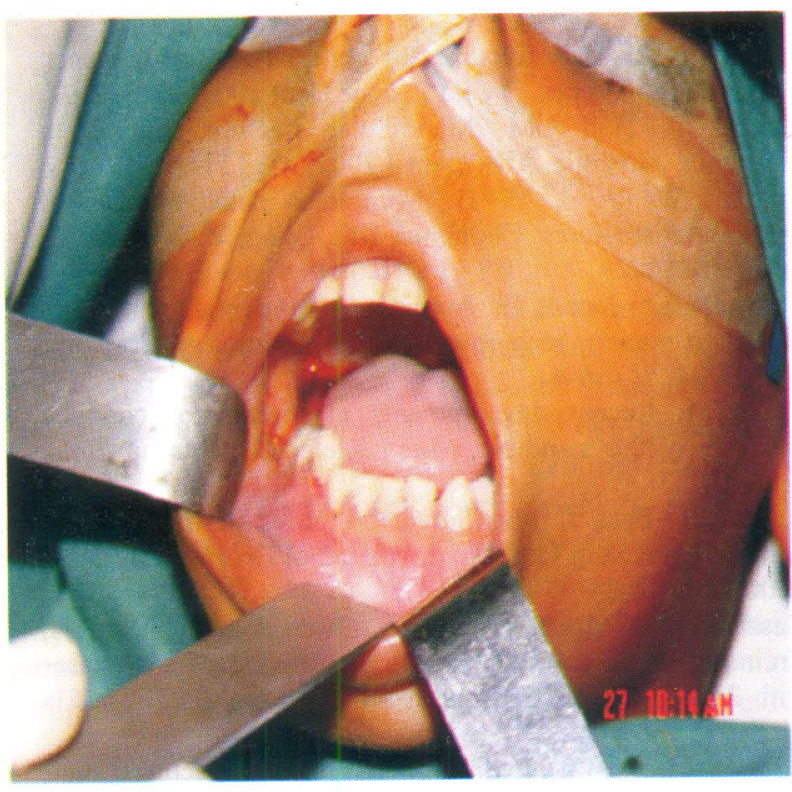

Fig. 1: Fracture mandible at deciduous right canine area (parasymphysis).

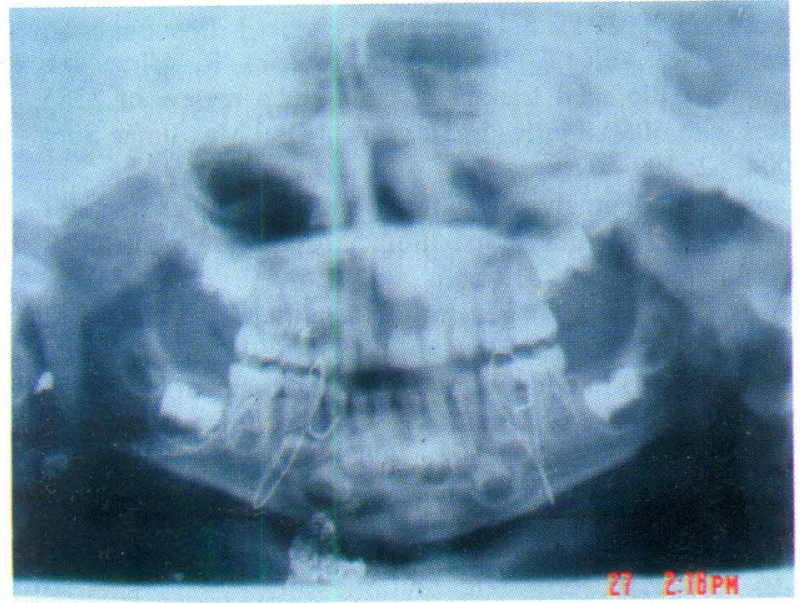

Fig. 3: Postoperative orthopantomograph with circummandibular wiring of splint.

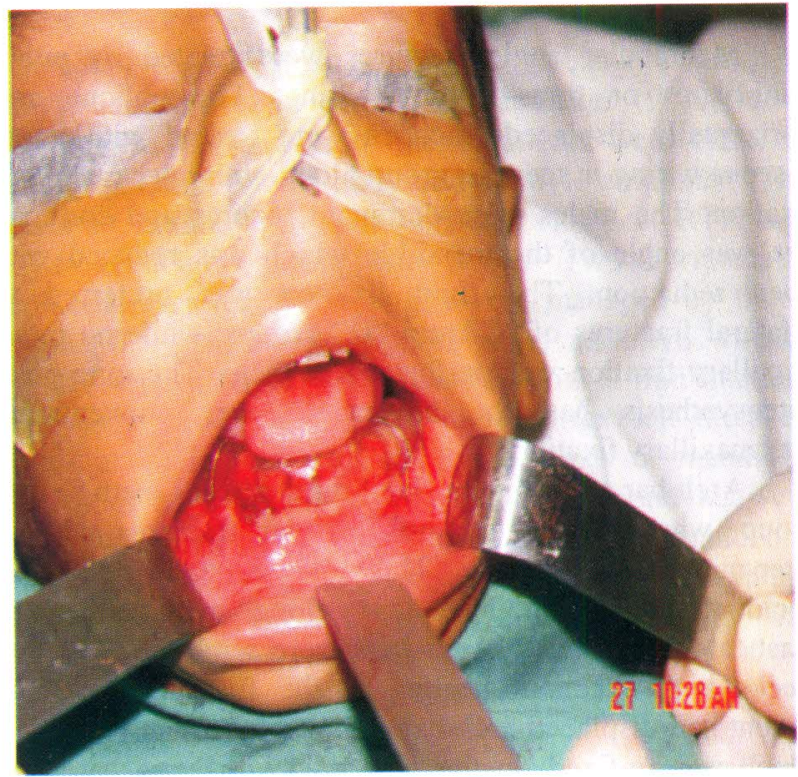

Fig. 2: Fracture mandible treated using acrylic splint with circummandibular wires.

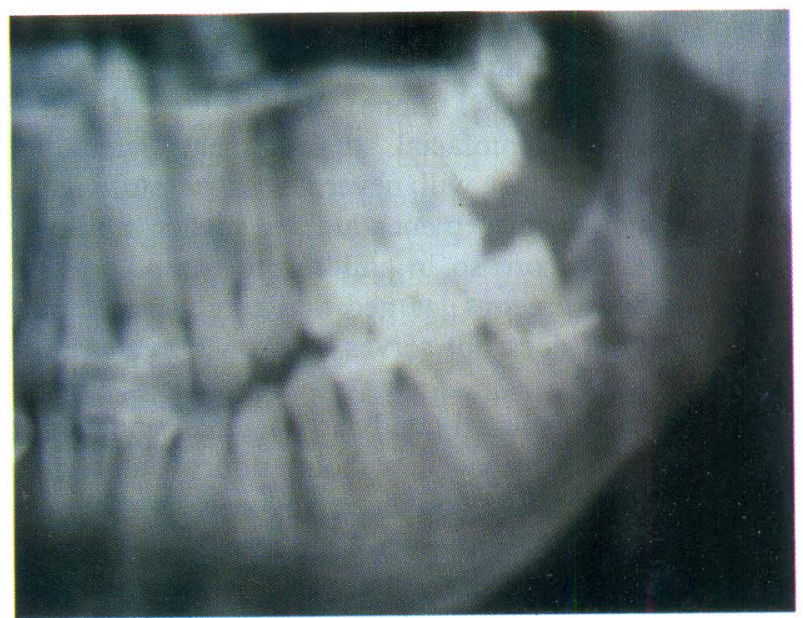

Fig. 4: Postoperative orthopantomograph of patient showing Transosseous wiring. 


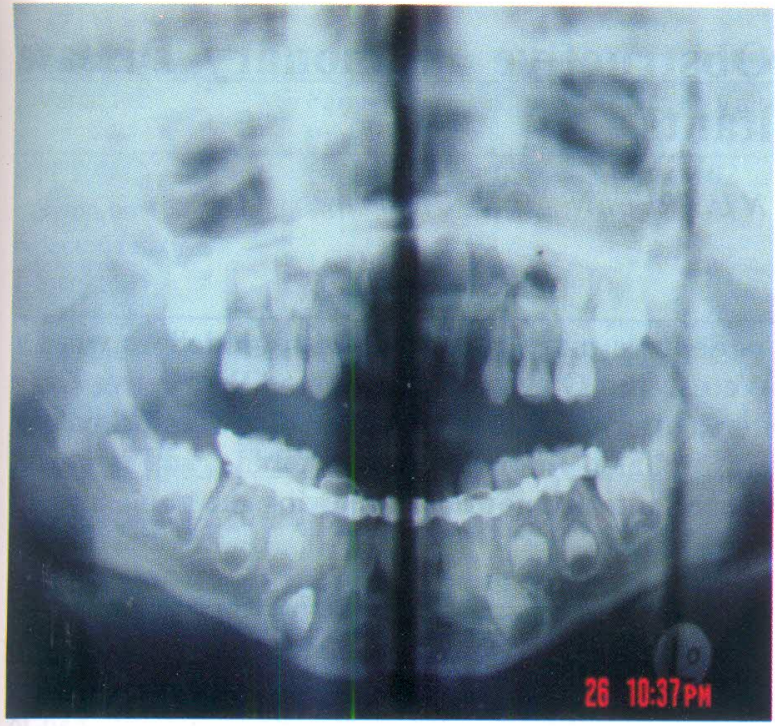

Fig 5: Fracture mandible treated with Eric arch bar without intermaxillary fixation.

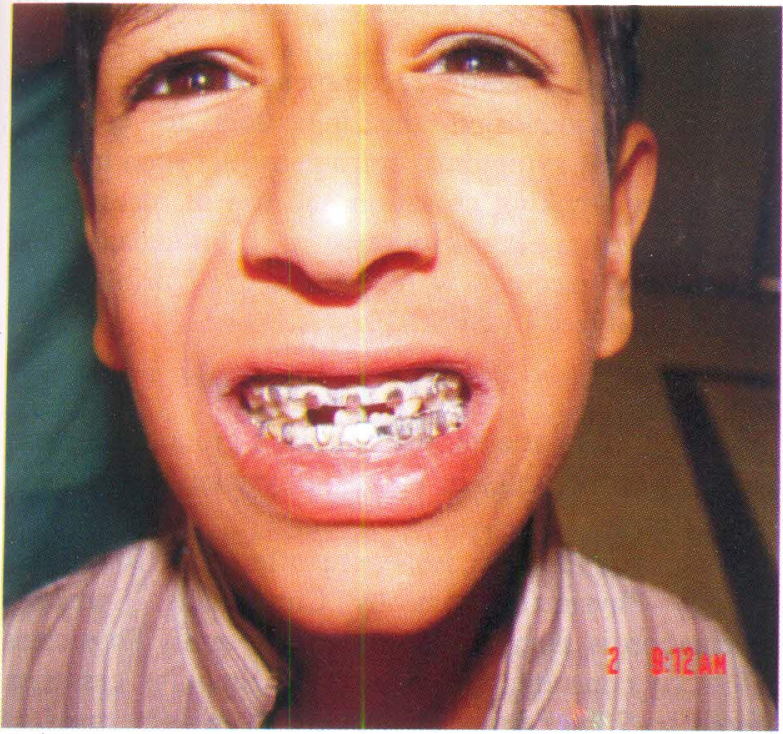

Fig. 7: Fracture mandible treated with Eric arch bar wiring $\&$ intermaxillary fixation.

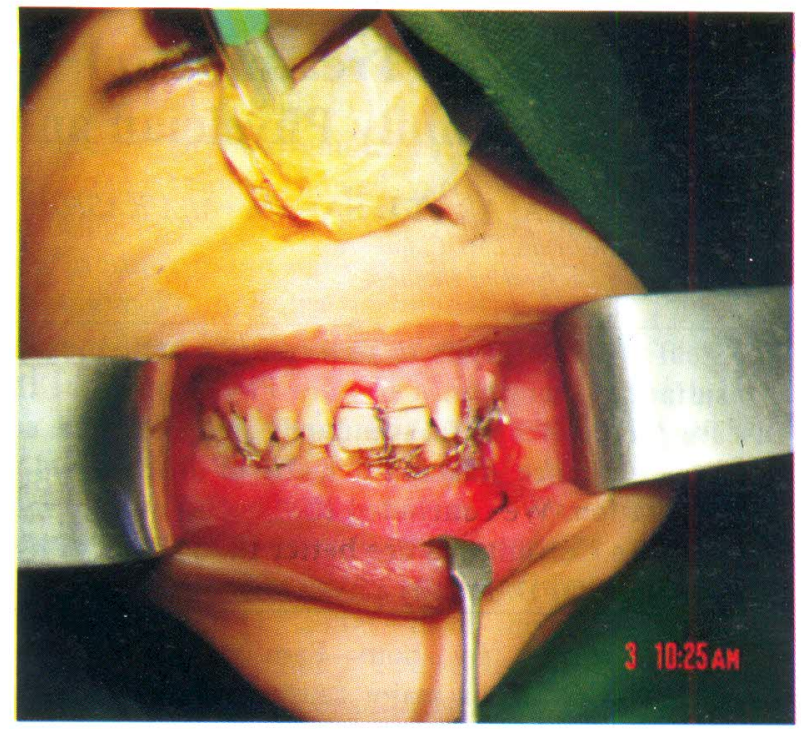

Fig. 6: Fracture mandible treated with Ivy eyelet wiring \& intermaxillary fixation.

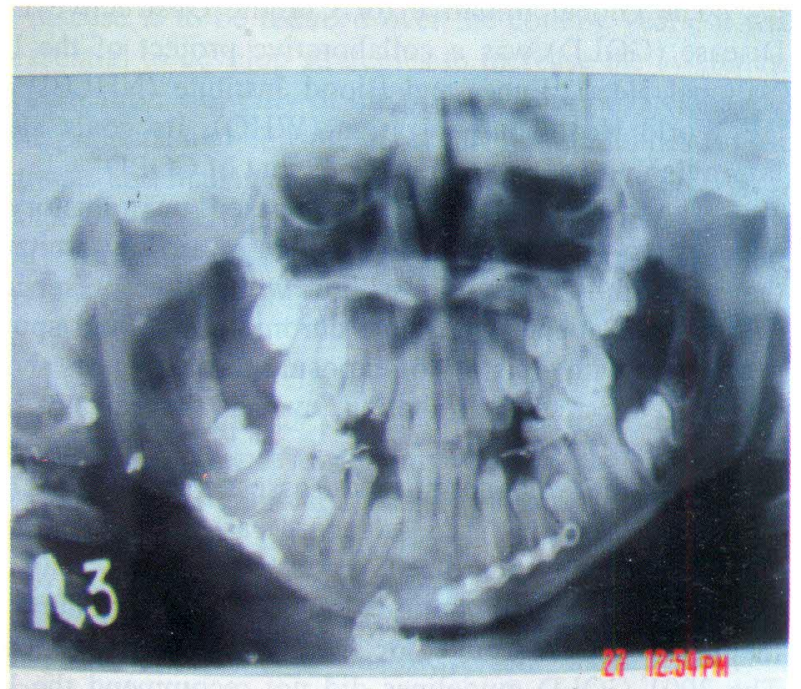

Fig. 8: Fracture mandible treated with titanium miniplate osteosynthesis. 